Empowering Anxious Parents to Manage Child Avoidance Behaviors: Randomized Control Trial of a Single-Session Intervention for Parent Accommodation

Jenna Y. Sung, B.A.*, Emma E. Mumper, Ph.D.*, Jessica L. Schleider, Ph.D. Department of Psychology, Stony Brook University

\title{
*Joint first-authorship
}

Corresponding author and primary contact for public and scientific inquiries about this manuscript: Jenna Sung, B.A; Jenna.Sung@stonybrook.edu; Tel: 631.632.7800; Stony Brook University

Disclosures. This project was funded by the Psi Chi Honor Society’s Graduate Research Grant. JYS receives funding from the Health Policy Research Scholars Program. JLS receives research support from the National Institutes of Health (DP5OD28123), the Klingenstein Third Generation Foundation, the American Psychological Foundation, the Upswing Fund, and Limbix, Inc. JLS is also a co-author of a therapeutic workbook for youth (published by New Harbinger Publications) and co-editing a book on low-intensity mental health interventions for youth with Oxford University Press. The authors report no other financial conflicts. 


\begin{abstract}
Background: A majority of youth who need anxiety treatment never access support. This disparity reflects a need for more accessible, scalable interventions_-particularly those that may prevent anxiety in high-risk children, mitigating future need for higher-intensity care. Selfguided single-session interventions (SSIs) may offer a promising path toward this goal, given their demonstrated clinical utility, potential for disseminability, and low-cost. However, existing self-guided SSIs have been designed for completion by adolescents already experiencing symptoms, and their potential for preventing anxiety in children-for instance, by mitigating known anxiety risk factors - remains unexplored. Objective: This trial evaluated the acceptability and proximal effects of Project EMPOWER: a web-based, self-guided SSI designed to reduce parent accommodation, a parenting behavior known to increase anxiety risk in offspring. Method: 301 parents reporting elevated anxiety symptoms with children ages 4-10 received either Project EMPOWER or an informational control (containing psychoeducational materials and resources); parents self-reported their accommodation of child anxiety and overall distress tolerance at baseline and 2-week follow-up. Results: Relative to control-group parents, parents who received Project EMPOWER reported significant reductions in their accommodation of child anxiety $\left(d_{s}=0.61, P<.001\right)$, as well as significant increases in their distress tolerance $\left(d_{s}=0.43, P<.001\right)$, from baseline to 2 -week follow-up. Additionally, parents rated Project EMPOWER as highly acceptable (e.g., easy to use, helpful, engaging) per preregistered benchmarks. Conclusions: Project EMPOWER is an acceptable self-guided SSI for parents of children at-risk for anxiety, yielding proximal reductions in clinically relevant targets.
\end{abstract} Keywords: Child and adolescent mental health, Prevention, Intervention, Digital Mental Health. Clinical Trials: NCT04453865; Analytic Code \& De-Identified Data: osf.io/z28p7/ 


\section{Introduction}

Anxiety disorders are among the most common, debilitating forms of childhood psychopathology, affecting $8.3-27.0 \%$ of youth before age 18 [1,2]. Child anxiety increases risk for psychiatric comorbidities across the lifespan [3], creates significant burdens for caregivers [4], and carries stark societal costs [5,6]. Although numerous interventions have been developed to treat youth anxiety disorders, up to $82.2 \%$ of US youth with anxiety will not receive adequate care [7]. Several factors may explain this discrepancy, including the length and cost of existing treatments and limited accessibility for families in need. Together, these factors create a pressing need for accessible, brief preventive programs to decrease odds of anxiety disorder onset in atrisk youth.

Single-session interventions (SSIs) may offer one potential solution to this gap in care. SSIs include core components of comprehensive evidence-based interventions delivered succinctly to improve the odds of access and completion [8]. In a meta-analysis of 50 randomized trials, SSIs reduced youth psychopathology across multiple disorders, with SSIs targeting child anxiety producing especially large effects (mean $g=0.58$; [8]). Thus, well-targeted SSIs may offer cost-effective additions or alternatives to traditional care for anxiety in youth. However, most existing SSIs for child anxiety target populations already experiencing clinical distress, leaving a need for options that may prevent anxiety in vulnerable children. Given that family factors play a crucial role in the etiology of child anxiety [1], SSIs targeting parents and their interactions with offspring may be a promising approach to preventing youth anxiety [9]. Thus, this trial examined the acceptability and short-term effects of a novel, web-based, selfguided SSI targeting parental accommodation: a well-established, potentially modifiable risk factor for child anxiety [10-13]. 


\section{Parental Accommodation as a Modifiable Intervention Target. Parent}

accommodation refers to changes in caregiver behaviors that facilitate or maintain their child's anxiety-driven avoidance behaviors $[14,15]$. Examples include modifying family routines (i.e., staying home from work to mitigate a child's separation fears) or directly participating in a child's avoidance strategies (i.e., keeping a child home from school). Parent accommodation reduces children's immediate distress but maintains their long-term avoidance of feared stimuli or situations, and high levels of parent accommodation are associated with more severe anxiety symptoms in offspring [15-17]. Parent accommodation may be further maintained by caregiverlevel factors, including elevated parental anxiety symptoms and low distress tolerance. For instance, accommodation behaviors are more frequent among parents reporting higher distress about their child's anxiety symptoms [18] and perceptions that experiencing anxiety is harmful to youth [19].

Parent accommodation can also be systematically reduced via psychosocial intervention. For instance, in trials of the 12-week, parent-directed, therapist-delivered Supportive Parenting for Anxious Childhood Emotions (SPACE) program, targeting parents' accommodation has helped mitigate child anxiety in children with subclinically and clinically-elevated anxiety symptoms $[15,20]$; recent studies even suggest that parent-directed, accommodation-focused treatments may be non-inferior to exposure therapy for treating child anxiety [21]. Translating core components of existing multi-session interventions for parental accommodation into briefer, self-guided SSIs (i.e., those that do not involve a trained therapist) may improve families' access to empirically driven supports. Thus, we developed and tested a web-based, self-guided SSI for parents - "Project EMPOWER" - designed to provide psychoeducation and teach skills to reduce parents' accommodation of avoidance behaviors in their school-aged children. Within an 
enhanced waitlist-control design, parents were randomized to either (a) Online Resources and Referrals (ORR) and Project EMPOWER, or (b) ORR and delayed Project EMPOWER access (after the study was complete, two weeks later). We predicted that parents would report larger declines in self-reported accommodation behaviors (primary outcome) and larger increases in distress tolerance (secondary outcome) in the ORR+EMPOWER group, relative to the ORR+waitlist, from baseline to 2 -week follow-up. We also predicted that parents completing Project EMPOWER would subjectively perceive larger pre-to immediate-post-SSI increases in their ability to help their child manage distressing situations, relative to control-group parents. Finally, we predicted that parents completing Project EMPOWER would rate it as acceptable (enjoyable, worth recommending to other parents, and personally helpful).

Notably, because this trial constituted the first formal test of Project EMPOWER, the study's primary goal was to assess the program's potential to engage its intended mechanistic target: Parent accommodation of avoidance and anxiety in their young children. If Project EMPOWER can systematically improve this target in parents of children both with and without clinically-elevated anxiety, this study may set the stage for future trials of Project EMPOWER's capacity to prevent child anxiety symptoms in the longer-term.

\section{Method}

Ethical Considerations. Study procedures were reviewed and approved by the University Institutional Review Board, and online consent was obtained from each participant, prior to participation. The trial and all methods were prospectively pre-registered in clincialtrials.gov prior to participant enrollment (NCT04453865). 
Recruitment and resulting sample. 301 parents of children ages 4-10 were recruited online via Facebook advertisements, following established ethics guidelines for passive, social media-based study recruitment [22]. Participants were eligible for the study if they (1) reported subclinical or greater anxiety symptoms (a score of $\geq 40$ on the Penn State Worry Questionnaire-PSWQ), because children with higher-anxiety parents are at elevated risk for developing anxiety themselves, and higher-anxiety parents report engaging in more accommodation than do lower-anxiety parents [17]; (2) had at least one child between 4-10 years old; and (3) endorsed comfort with English (intervention materials were available in English only). This specific child age-range was selected because it encompasses the age of onset for common child anxiety disorders [23]; it also matches the age-range for which parent-focused interventions are often designed [24]. Study recruitment began July 2020 and ended in August of 2020 once the target $\mathrm{N}$ was reached.

Procedures. After clicking on a social media-based advertisement, parents were directed to an informational study webpage inviting them to complete an online eligibility screener. Eligible parents then reviewed an online consent form inviting them to participate. Parents could initiate the study at any time and location, using any internet-equipped device (smartphone, laptop, tablet). After starting the study, participants first completed pre-intervention self-report questionnaires, detailed below. Within the same survey, participants were randomized via Qualtrics (1:1 allocation ratio) to either receive Online Resources and Referrals (ORR) and immediate access to Project EMPOWER (intervention condition), or ORR and delayed Project EMPOWER access after the two-week follow-up (control condition). Those in the intervention condition also completed the Program Feedback Scale, along with other post-intervention surveys, immediately following Project EMPOWER completion. Two weeks later, all parents- 
regardless of condition - were invited to complete follow up questionnaires. Parents in the control condition were then invited to complete Project EMPOWER, if they were interested in doing so (completion of Project EMPOWER subsequent to follow-up questionnaires was optional, not part of the study). Thus, all participants were able to complete Project EMPOWERs, either immediately or after a 2-week delay.

Intervention. Project EMPOWER (freely available for anonymous completion at www.schleiderlab.org/EMPOWER) is a web-based, self-guided SSI for parents that takes 20-30 minutes to complete. The program includes five main elements, based on current recommended practices in SSI design [28] and existing, therapist-delivered interventions targeting parental accommodation $[15,21]$ :

1. Psychoeducation on child anxiety and avoidance, along with how parental accommodation can inadvertently maintain child anxiety

2. Information on how parents can better identify children's patterns of avoidance and encourage "brave behavior," instead

3. An exercise guiding parents in creating a personalized, step-by-step "action plan" for promoting brave, approach-oriented behaviors (rather than anxiety-driven avoidance) in their own child

4. A segment intended to normalize parent distress responses in response to anxiety in offspring, including a rationale for why encouraging "brave behaviors"—-despite being emotionally challenging for caregivers —ultimately bolsters children's well-being and resilience

5. A vignette exercise in which parents read about another family's difficulty managing their child's anxiety; parents identify various elements of the "anxiety cycle" (per 
psychoeducation provided previously) and generate possible solutions for the parents described in the vignette, based on their newfound knowledge of promoting "brave behavior" in youth.

Control condition. Online Resources and Referrals (ORR) included an information sheet containing a list of web-based psychoeducational resources (videos, books, online toolkits, etc.) on anxiety, hotlines, and resources on finding mental health treatment around the U.S. ORR did not include any psychoeducational components explicitly designed to reduce parental accommodation of child anxiety. For the full content of ORR, reference Appendix A.

Measures. Other measures not detailed here were included in the study for exploratory purposes. Full battery of measures included in the study can be found on the clinicaltrials.gov registration.

Demographics. Parents self-reported gender, biological sex, ethnicity, country of origin, education level, and age for themselves and their child.

Parent Accommodation of Child Anxiety (Primary outcome). Via the Family Accommodation Scale-Anxiety (FASA; [10]). Parents rated agreement with 9-items reflecting the extent to which they accommodate their child's anxiety symptoms and/or avoidance behaviors. Higher mean scores indicate more frequent parental accommodation. As a primary outcome measure, the FASA was administered at baseline and 2-week follow up to all participants. The FASA has demonstrated excellent psychometric properties across numerous studies (Lebowitz et al., 2013). Here, alphas were 0.87 and 0.85 at baseline and 2-week followup, respectively.

Parent Distress Tolerance (Secondary outcome). Via the 16-item Distress Tolerance Scale (DTS; [25]) — a valid, reliable measure of overall distress tolerance in adults_-parents 
rated on a 5-point scale their perceived ability to experience and withstand distressing emotional states. Higher mean DTS scores reflect lower levels of distress tolerance. As a secondary outcome measure, DTS was administered at baseline and 2-week follow to all study participants. Here, alphas were 0.86 and 0.88 at baseline and 2-week follow-up, respectively.

Child Anxiety Symptoms. Parents completed Revised Children's Anxiety and Depression Scale-Parent Report (RCADS-25-P ;[26]): a well-validated, 25-item measure assessing child internalizing symptoms. Parents endorsed the presence (or absence) of 25 different anxiety and depressive symptoms in children, each on a 4-point scale. Higher scores reflect more severe child internalizing symptomatology. The RCADS-25-P was completed at baseline only to characterize the level of anxiety experienced by children of participating parents. Alpha for the RCADS-25 was 0.85 in this study. Notably, we did not assess child anxiety at 2week follow-up in this study, because the trial's objective was to establish whether Project EMPOWER could successfully engage its intended target (parent accommodation behaviors).

Parent Anxiety Symptoms. Parents completed the Penn State Worry Questionnaire (PSWQ; [27]), a well validated 16-item self-report questionnaire asking respondents to rate their perceived experience of worry- and anxiety-related problems, using a 5-point scale. Higher sum scores indicate more severe worry. Parents completed the PSWQ at baseline to screen for subclinical or higher parental anxiety levels (an inclusion criterion) and to characterize the participating parent sample by anxiety severity. The PSWQ has demonstrated high internal consistency and good test-retest reliability [27]. Alpha for the PSWQ was 0.88 in this study.

Perceived change in preparedness to help child manage distress. A single-item measure gauging participants' perceived changes in their ability to help their children manage distressing situations was adapted for this study [28]. All participants were asked to rate 
agreement on a 5-point scale with a single-item statement, either immediately after completing Project EMPOWER (intervention condition) or immediately after being presented with psychoeducational materials (informational waitlist condition): “compared to before you started this survey, how prepared do you feel to help your child manage distressing situations?" This item was administered immediately-post SSI only for the intervention group as a secondary, exploratory outcome.

Intervention Acceptability. Parents in the intervention condition completed the Program Feedback Scale (PFS; [29]), a reliable and valid measure routinely used to assess acceptability and user perceptions of web-based, self-guided SSIs. The PFS asks participants to rate 7 statements on a 1-5 scale and share what they (a) liked and (b) would change about the SSI, in an open-response format. A mean score of 3 or higher indicates acceptability and positive program evaluation. The PFS was administered at post-SSI to parents assigned to the intervention condition to assess program acceptability.

Power Analysis. Using G*Power 3.1, sample sizes needed to detect group differences in the primary outcome (changes in accommodation from baseline to follow-up) between the intervention and control groups of small (.2), medium (.5), and large effects (.8) based on an $F$ test, linear multiple regression with $\alpha=.05$ and power $=0.80$, were 395,55 , and 25 , respectively. Thus, our sample $(\mathrm{N}=301)$ offered sufficient power to detect a small-to-medium effect betweengroups effect (consistent with effect sizes observed in previous randomized trials of online SSIs [30]).

Missing Data. We imputed missing data using the expectation-maximization and bootstrapping algorithm implemented with Amelia II in R, as no evidence emerged for unequal drop-out by condition. These imputed datasets allowed for more conservative intent-to-treat 
analyses than listwise deletion or last-observation carried forward and allowed us to retain high power even in the presence of missing data. We imputed 60 datasets, per the percent of missing data for our primary outcome measure (FASA) at 2-week follow-up.

\section{Analytic Plan.}

The full pre-registration can be found on ClinicalTrials.gov (CT04453865). De-

identified data and code for all pre-registered analyses are available on Open Science Framework (https://osf.io/s5wua/).

\section{Project EMPOWER effects on primary and secondary outcomes. To assess} intervention effects on parent's accommodation levels and distress tolerance from baseline to 2week follow up, we ran a multiple linear regression with the intervention condition $(1=\mathrm{ORR}+$ Project EMPOWER; $0=\mathrm{ORR}+$ waitlist $)$, baseline accommodation levels, and parental distress as predictor variables to examine whether participants in each condition saw a differential reduction in outcome variables. Using the MOTE R Package, we also reported Cohen's $d$ effect sizes and 95\% confidence intervals for within-group ( $d$ _av; reflecting intervention effects for pre-to-follow-up change) and between-group ( $d$ _s; reflecting changes in outcome from pre-to-follow-up in the two groups) for both accommodation and distress tolerance levels [31].

\section{Perceived change in preparedness to manage child anxiety from pre- to post-survey.} A two-sample $t$-test determined whether the overall, subjectively-detectable pre-to-post changes in "preparedness to help their child manage anxiety" significantly differed between parents who completed Project EMPOWER immediately, versus control-group parents.

Intervention Acceptability. We examined overall and item-level mean PFS scores among parents who completed Project EMPOWER. Mean and item-level scores of $\geq 3$ or higher 
on any item (on a 1-5 scale) reflected endorsement of the program's acceptability (e.g. positive feedback), either for that specific item or overall.

Project EMPOWER completion rates. Operational definitions of differential "program completer" status among parents assigned to the Project EMPOWER condition were preregistered prior to data analysis. Full completers were those they reached the final page of the intervention, thus receiving the full "dose" of intended materials (approximate completion time: 25-30 minutes); personalized plan completers completed all psychoeducational content in Project EMPOWER and finished their personalized plan for promoting brave behavior in their child (approximate completion time: 20-25 minutes); psychoeducational content completers completed all psychoeducational content, but not a personalized plan (approximate completion time: 10-15 minutes); and partial completers began the intervention but did not reach any of the above-mentioned program benchmarks. We report completion rates at each level, among parents assigned to the Project EMPOWER condition, in the CONSORT diagram (Figure 1). 
Figure 1. CONSORT Diagram

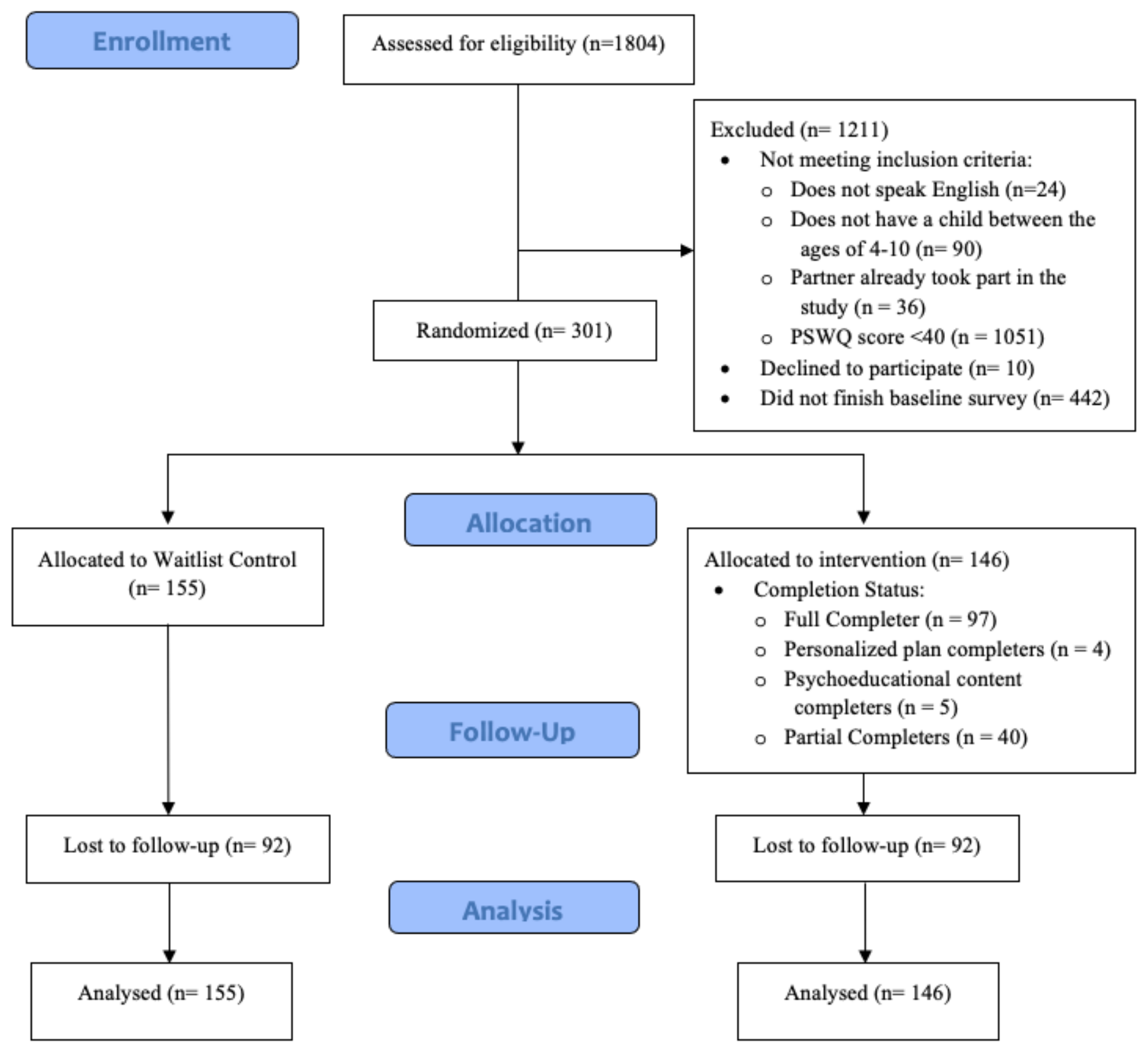

\section{Results}

Sample Characteristics. Characteristics of the 301 participating parents and their children are displayed in Table 1 . Parents were predominantly female (98.00\%), 67.77\% White, 
9.63\% Asian, 9.63\% other, 6.31\% Hispanic/Latino/a, 2.33\% American Indian/Alaska Native, 1.99\% More than one race, 1.66\% Black/African American, and 0.66\% Native Hawaiian/Pacific Islander; $52.82 \%$ had a received a graduate or professional degree. Consistent with our goal to recruit parents with elevated levels of anxiety, participants reported a mean score of 60.74 $(S D=9.84)$ on the PSWQ, just below the clinical cut-off score of 62, per Behar and colleagues [32]. Parents indicated a broad range of difficulties facing their child ( $M_{\text {raw } R C A D S}$ score $=18.83$, $S D_{\text {raw } R C A D S}$ score $\left.=9.95\right)$, including: clinically significant anxiety symptoms $(76.74 \%)$, mood problems (17.94\%), behavioral problems (33.89\%), attention problems (33.89\%), developmental delay (6.31\%), learning disabilities (7.31\%), peer relationships (35.55\%), family relationship problems $(21.93 \%)$, and trauma $(15.28 \%)$.

Table 1. Sample Characteristics

\begin{tabular}{lcc}
\multicolumn{1}{c}{ Variable } & $\begin{array}{c}\text { Project EMPOWER } \\
(\mathrm{n}=146)\end{array}$ & $\begin{array}{c}\text { Waitlist Control } \\
(\mathrm{n}=155)\end{array}$ \\
\hline Parent PSWQ (M/SD) & $60.30(9.81)$ & $61.18(9.89)$ \\
Youth RCADS (M/SD) & $18.08(9.64)$ & $19.53(10.22)$ \\
Youth Age (M/SD) & $6.77(1.93)$ & $6.73(2.03)$ \\
Youth Gender (N/\% Female) & $75(51.02 \%)$ & $67(43.51 \%)$ \\
Youth Race/Ethnicity (N/\%) & & $5(3.23 \%)$ \\
American Indian/Alaska Native & $5(3.42 \%)$ & $11(7.10 \%)$ \\
Asian & $11(7.53 \%)$ & $1(0.65 \%)$ \\
Black/African American & $4(2.74 \%)$ & $8(5.16 \%)$ \\
Hispanic/Latino/a & $6(4.11 \%)$ & $101(65.16 \%)$ \\
White/Non-Hispanic & $87(59.59 \%)$ & $19(12.26 \%)$ \\
$>1$ Race & $14(9.59 \%)$ &
\end{tabular}


Other

Family Annual Income (N/\%)

$\$ 0-\$ 19,000$

$\$ 20,000-\$ 39,000$

$\$ 40,000-\$ 59,000$

$\$ 60,000-\$ 79,000$

$\$ 80,000-\$ 99,000$

$\$ 100,000-\$ 119,000$

$\$ 120,000-\$ 140,000$

$\$ 140,000+$

Unknown

Marital Status

Married

Living with Partner

Never Married

Divorced

Separated

Widowed

Single Parent

$\mathrm{N}$ children $(\mathrm{M} / \mathrm{SD})$

Parent Sex (N/\% Female)
$19(13.01 \%)$

$10(6.45 \%)$

$\begin{array}{cc}9(6.16 \%) & 8(5.16 \%) \\ 16(10.96 \%) & 20(12.90 \%) \\ 18(12.33 \%) & 12(7.74 \%) \\ 16(10.96 \%) & 18(11.61 \%) \\ 16(10.96 \%) & 15(9.68 \%) \\ 11(7.53 \%) & 14(9.03 \%) \\ 15(10.27 \%) & 13(8.39 \%) \\ 27(18.49 \%) & 31(20.00 \%) \\ 18(12.33 \%) & 24(15.48 \%)\end{array}$

$104(71.23 \%)$

$118(76.13 \%)$

$14(9.59 \%)$

$12(7.74 \%)$

$13(8.90 \%)$

$10(6.45 \%)$

$9(6.16 \%)$

$8(5.16 \%)$

$4(2.74 \%)$

$6(3.87 \%)$

$2(1.37 \%)$

$1(0.65 \%)$

$32(21.92 \%)$

$32(20.65 \%)$

$2.02(1.00)$

2.19 (1.20)

$143(97.95 \%)$
$152(98.06 \%)$

\section{Did Project EMPOWER reduce parent accommodation of anxiety and improve}

parent distress tolerance? Parents assigned to the Project EMPOWER condition reported significantly greater reductions in accommodation of their children's anxiety, between-group $d_{\mathrm{s}}=$ $0.61, P<.001)$, as well as significantly greater improvements in distress tolerance $\left(d_{\mathrm{av}}=0.17\right.$; 
between-group $d_{\mathrm{s}}=0.43, P<.001$ ) from baseline to 2-week follow-up, relative to control-group parents. Refer to table 2 for additional details of the multiple linear regression.

Table 2. Results of multiple linear regressions predicting intervention effects on parent accommodation (FASA) and distress tolerance (DTS) at 2-week follow-up.

\begin{tabular}{|c|c|c|c|c|c|c|}
\hline & \multicolumn{3}{|c|}{ Parent-reported accommodation } & \multicolumn{3}{|c|}{ Parent reported distress tolerance } \\
\hline & $B$ & $S E$ & $P$ & $B$ & $S E$ & $P$ \\
\hline $\begin{array}{l}\text { FASA } \\
\text { Baseline }\end{array}$ & 0.53 & 0.07 & $<.001$ & & & \\
\hline $\begin{array}{l}\text { DTS } \\
\text { Baseline }\end{array}$ & & & & 0.77 & 0.07 & $<.001$ \\
\hline Intercept & 0.79 & 0.15 & $<.001$ & 0.75 & 0.20 & $<.001$ \\
\hline Condition & -0.48 & 0.11 & $<.001$ & -0.24 & 0.09 & .008 \\
\hline
\end{tabular}

Regarding within-group effects, parents who received Project EMPOWER reported significant two-week reductions in accommodation of child anxiety (Project EMPOWER withingroup $d_{\mathrm{av}}=0.67$ ), whereas parents assigned to the control condition did not (control within-group $\left.d_{\mathrm{av}}=0.17\right)$. Between and within-group effect sizes $\left(d_{\mathrm{av}}\right.$ and $\left.d_{\mathrm{s}}\right)$ and $95 \%$ confidence intervals are reported in Table 3 .

Table 3. Means, standard deviations, and effect sizes of outcome variables by condition

\begin{tabular}{cccccccc}
\hline & \multicolumn{2}{c}{ Project EMPOWER } & & \multicolumn{2}{c}{ Control Group } & & \\
\cline { 2 - 3 } $\begin{array}{c}\text { Outcome } \\
\text { Variable }\end{array}$ & $\begin{array}{c}\text { M(SD) } \\
\text { Baseline }\end{array}$ & $\begin{array}{c}\text { M (SD) } \\
\text { 2-week } \\
\text { follow-up }\end{array}$ & $\begin{array}{c}\text { Cohen's d_av } \\
{[95 \% \text { CI }]}\end{array}$ & $\begin{array}{c}\text { M (SD) } \\
\text { Baseline }\end{array}$ & $\begin{array}{c}\text { M (SD) } \\
\text { 2-week } \\
\text { follow-up }\end{array}$ & $\begin{array}{c}\text { Cohen's d_av } \\
{[95 \% \text { CI }]}\end{array}$ & $\begin{array}{c}\text { Cohen's } \\
\text { d_s } \\
{[95 \% \text { CI }]}\end{array}$ \\
\hline FASA & 1.83 & 1.29 & 0.67 & 1.88 & 1.79 & 0.11 & 0.61 \\
& $(0.91)$ & $(0.71)$ & {$[0.49,0.85]$} & $(0.90)$ & $(0.81)$ & {$[-0.05,0.26]$} & {$[0.38,0.84]$}
\end{tabular}


DTS

2.77

$(0.76)$
2.64

$(0.73)$
0.17

$[0.01,0.34]$
2.73

$(0.72)$
2.85

$(0.76)$
$-0.16$

0.43

$[-0.32,0.00]$

Note. Cohen's $d$ _av reflects within-group change in each outcome variable; Cohen's $d$ s reflects betweengroup change in each outcome.

\section{Did parents who completed Project EMPOWER perceive improvements in their} preparedness to manage child distress? Immediately following completion of either the control condition or Project EMPOWER, participants were asked: "compared to before you started this survey, how prepared do you feel to help your child manage distressing situations?" On a scale of 1 (Much less prepared to help my child) to 5 (A lot more prepared to help my child), parents who completed Project EMPOWER endorsed feeling significantly more prepared to help their child compared control-group parents, $t(155.27)=8.66, P<.001$. Among parents who received immediate access to project EMPOWER and completed the intervention, $54.28 \%$ reported feeling "a little more prepared to help my child", 30.00\% reported feeling "a lot more prepared", and $15.71 \%$ reported feeling “the same amount prepared." No participant reported feeling less prepared to help their child.

Was Project EMPOWER Acceptable? Among parents assigned to the intervention condition, the majority ( $N=97,66.44 \%)$ fully completed project EMPOWER; $2.74 \%(\mathrm{~N}=4)$ qualified as "personalized plan completers," 3.42\% (N=5) were "psychoeducation content completers," 21.92\% (N=32) were "partial completers," and the remainder (5.48\%) did not begin Project EMPOWER following randomization. Parents who completed project EMPOWER rated the intervention as acceptable per a mean PFS score of 4.25 out of 5 (higher than the preregistered cut-off score of 3). More specifically, parents rated the intervention as easy to understand $(M=4.41 / 5.00)$, easy to use $(M=4.31 / 5.00)$, likely to help other parents 
$(M=4.31 / 5.00)$, enjoyable $(M=3.92 / 5.00)$, worth recommending to other parents $(M=4.20 / 5.00)$, and endorsed agreement with the program's message $(M=4.56 / 5.00)$

\section{Discussion}

Trial results support the short-term efficacy and acceptability of Project EMPOWER: a self-guided, online single-session intervention designed to reduce parental accommodation of child anxiety. Compared to a psychoeducational control, Project EMPOWER yielded significant reductions in clinically-relevant outcomes - parental accommodation and distress toleranceacross a two-week follow-up period. Additionally, parent participants viewed Project EMPOWER as highly acceptable and subjectively helpful for managing their child's distress relative to the psychoeducational control. Moreover, parents who began Project EMPOWER completed the program at a relatively high rate $(66.44 \%)$, both when compared to a prior naturalistic program evaluation of online, self-guided SSIs (e.g., 34.32\% completion rates for 3 other online SSIs; [28]) and compared to completion rates reported for similar self-guided, digital mental health supports $(0.5 \%-28.6 \%$; [33]). This retention level within a self-guided program suggests Project EMPOWER's strong acceptability among its users. Together, results suggest Project EMPOWER's promise to mitigate known risk factors for anxiety in children, and specifically those with higher-anxiety parents.

Notably, Project EMPOWER's between-group effects on parental accommodation ( $d$ FASA $=0.61)$ compared favorably to effects observed in separate trials of treatments targeting parent accommodation - including those observed in a trial of a 12-week, parent-directed, therapistguided intervention (the SPACE Program; Lebowitz et al., post-intervention $d$ FASA $=0.22$, versus child-directed exposure therapy). Project EMPOWER's effect sizes also compare favorably to previously-reported effects of SSIs targeting youth anxiety directly (post-SSI $d$ child anxiety $=0.56$; 
[8]. These previously-observed effects serve as approximate benchmarks for Project

EMPOWER's impact, rather than direct comparisons, due to variation in methods (e.g., more versus less active comparison groups), follow-up length, and intervention intensity. Nonetheless, present results are the first to suggest that a 30-minute, fully self-guided, parent-directed intervention may help reduce parental accommodation, potentially helping to mitigate anxiety in their children. Frequently-cited benefits of self-guided, online SSIs - including their potential for rapid scalability, their free availability to users, and that they may be completed at any time and location (Schleider et al., 2020)—position Project EMPOWER for high potential public health impact, reaching individuals and populations who may be otherwise unable to access support.

Several trial limitations warrant discussion and suggest directions for future research. First, although the fully-online study design allowed a large sample size and rapid, low-cost recruitment via social media, the lack of monetary compensation likely contributed to substantial attrition at follow-up (61.13\%), despite scheduled email reminders. However, it is worth noting that offering greater monetary compensation may have introduced additional bias to the sample selection. This limitation was addressed via a rigorous missing data approach that has shown utility with high rates of missing data, inclusive of those observed here [34]. Second, similar to the limitations noted in much of the parenting intervention literature, our homogeneous sample with respect to sex ( $98 \%$ mothers), race/ethnicity, and education status, limited the generalizability of our results across diverse groups of parents. This may be due to the selection bias introduced by the social media recruitment as Facebook likely distributed the advertisements to users who are interested in the study topic. As the study team did not have control over the algorithms used to distribute the advertisement, it limited our ability to reach a more diverse population. Moving forward, it will be critical to test the acceptability and effects of Project 
EMPOWER and other self-guided SSIs among members of marginalized and minoritized communities who are systematically least likely to access traditional, face-to-face mental health treatments due to financial, logistical, and stigma-related barriers.

Given that non-English-speaking parents were unable to take part in the study (Project EMPOWER is currently available only in English), efforts to translate project EMPOWER into various languages may greatly facilitate tests of its acceptability and utility in more diverse caregivers. Third, because this trial was the first to test Project EMPOWER's acceptability and proximal effects, we included a relatively brief, two-week follow-up period. Thus, results speak only to the short-term effects of the intervention on known risk factors for child anxiety. Given that some trials of self-guided SSIs have demonstrated clinical benefits for youth up to 9 months post-intervention [30,35], longer-term effects of Project EMPOWER remain important to explore. Such studies may assess whether the intervention can prevent the emergence of child anxiety symptoms and evaluate improvements in parent accommodation and distress tolerance as possible change mechanisms.

In sum, Project EMPOWER shows promise as a scalable, brief, self-guided approach to reducing accommodation behaviors and strengthening distress tolerance among high-anxiety parents of school-aged children — at least in the short-term. Future research should examine whether and how Project EMPOWER may be useful as both a stand-alone intervention (as assessed here) and as a possible adjunctive support in the context of longer-term, child-directed anxiety treatment, for families in need of more intensive clinical support. For example, clinicians may assign project EMPOWER as "homework" to augment traditional psychoeducation about the nature and maintenance of anxiety; alternatively, therapists might deploy Project EMPOWER as a relapse prevention tool, introduced at termination of child-focused treatment, or as an 
interim support for families on waiting-lists for treatment. Future work may gauge Project EMPOWER's potential as a therapy-augmenting tool and evaluate its impacts on treatment duration and youth symptom change. Given project EMPOWER's potential for rapid scalability (as a free, self-guided, online intervention), future work should also test its possible use across multiple settings and by diverse populations. This initial trial recruited parents of young children; however, Project EMPOWER teaches skills of potential relevance to any adult who interacts with children - including teachers, mentors, and healthcare workers. Thus, Project EMPOWER may be integrated into youths' many existing environments via direct distribution to adults who care for them, who may utilize the program however and wherever they choose.

Acknowledgements. The authors thank Michael Mullarkey for his guidance in dataanalysis. The authors also thank Akash Shroff and Julia Fassler for assistance with recruitment, data management, and technical support throughout the study.

The first and senior authors of this manuscript could not have accomplished this work without the collaboration, creativity, dedication, and leadership of their talented co-author, Emma Mumper, Ph.D. Dr. Mumper co-developed Project EMPOWER with Ms. Sung and Dr. Schleider; she was instrumental in the conceptualization and execution of this trial. This manuscript is dedicated to her memory. 


\section{References}

1. Schleider JL, Weisz JR. Family process and youth internalizing problems: A triadic model of etiology and intervention. Dev Psychopathol 2017 Feb;29(1):273-301. PMID:27048767

2. Costello EJ, Egger HL, Angold A. The developmental epidemiology of anxiety disorders: phenomenology, prevalence, and comorbidity. Child Adolesc Psychiatr Clin N Am 2005 Oct;14(4):631-48, vii. PMID:16171696

3. Copeland WE, Angold A, Shanahan L, Costello EJ. Longitudinal patterns of anxiety from childhood to adulthood: the Great Smoky Mountains Study. J Am Acad Child Adolesc Psychiatry 2014 Jan;53(1):21-33. PMID:24342383

4. Ramos-Cerqueira AT de A, Torres AR, Torresan RC, Negreiros APM, Vitorino CN. Emotional burden in caregivers of patients with obsessive-compulsive disorder. Depress Anxiety 2008;25(12):1020-1027. PMID:18833578

5. Beecham J. Annual Research Review: Child and adolescent mental health interventions: a review of progress in economic studies across different disorders [Internet]. Journal of Child Psychology and Psychiatry. 2014. p. 714-732. [doi: 10.1111/jcpp.12216]

6. Bodden DHM, Dirksen CD, Bögels SM. Societal burden of clinically anxious youth referred for treatment: a cost-of-illness study. J Abnorm Child Psychol 2008 May;36(4):487-497. PMID:18214667

7. Merikangas KR, He J-P, Burstein M, Swendsen J, Avenevoli S, Case B, Georgiades K, Heaton L, Swanson S, Olfson M. Service Utilization for Lifetime Mental Disorders in U.S. Adolescents: Results of the National Comorbidity Survey-Adolescent Supplement (NCS-A) [Internet]. Journal of the American Academy of Child \& Adolescent Psychiatry. 2011. p. 32-45. [doi: 10.1016/j.jaac.2010.10.006]

8. Schleider JL, Weisz JR. Little Treatments, Promising Effects? Meta-Analysis of SingleSession Interventions for Youth Psychiatric Problems. J Am Acad Child Adolesc Psychiatry 2017 Feb; 56(2):107-115. PMID:28117056

9. Degnan KA, Almas AN, Fox NA. Temperament and the environment in the etiology of childhood anxiety. J Child Psychol Psychiatry 2010 Apr; 51(4):497-517. PMID:20158575

10. Lebowitz ER, Woolston J, Bar-Haim Y, Calvocoressi L, Dauser C, Warnick E, Scahill L, Chakir AR, Shechner T, Hermes H, Vitulano LA, King RA, Leckman JF. FAMILY ACCOMMODATION IN PEDIATRIC ANXIETY DISORDERS [Internet]. Depression and Anxiety. 2013. p. 47-54. [doi: 10.1002/da.21998]

11. Lebowitz ER, Scharfstein LA, Jones J. Comparing family accommodation in pediatric obsessive-compulsive disorder, anxiety disorders, and nonanxious children. Depress Anxiety 2014 Dec;31(12):1018-1025. PMID:24677578 
12. Peris TS, Lindsey Bergman R, Langley A, Chang S, Mccracken JT, Piacentini J. Correlates of Accommodation of Pediatric Obsessive-Compulsive Disorder: Parent, Child, and Family Characteristics [Internet]. Journal of the American Academy of Child \& Adolescent Psychiatry. 2008. p. 1173-1181. [doi: 10.1097/chi.0b013e3181825a91]

13. Storch EA, Geffken GR, Merlo LJ, Jacob ML, Murphy TK, Goodman WK, Larson MJ, Fernandez M, Grabill K. Family accommodation in pediatric obsessive-compulsive disorder. J Clin Child Adolesc Psychol 2007 Apr;36(2):207-216. PMID:17484693

14. Lebowitz ER, Panza KE, Su J, Bloch MH. Family accommodation in obsessive-compulsive disorder. Expert Rev Neurother 2012 Feb;12(2):229-238. PMID:22288678

15. Lebowitz ER, Omer H, Hermes H, Scahill L. Parent Training for Childhood Anxiety Disorders: The SPACE Program. Cognitive and Behavioral Practice. 2014. p. 456-469. [doi: 10.1016/j.cbpra.2013.10.004]

16. Lebowitz ER, Scharfstein L, Jones J. Child-Report of Family Accommodation in Pediatric Anxiety Disorders: Comparison and Integration with Mother-Report. Child Psychiatry Hum Dev 2015 Aug;46(4):501-511. PMID:25209390

17. Thompson-Hollands J, Kerns CE, Pincus DB, Comer JS. Parental accommodation of child anxiety and related symptoms: range, impact, and correlates. J Anxiety Disord 2014 Dec;28(8):765-773. PMID:25261837

18. Storch EA, Lehmkuhl H, Pence SL, Geffken GR, Ricketts E, Storch JF, Murphy TK. Parental Experiences of Having a Child with Obsessive-Compulsive Disorder: Associations with Clinical Characteristics and Caregiver Adjustment [Internet]. Journal of Child and Family Studies. 2009. p. 249-258. [doi: 10.1007/s10826-008-9225-y]

19. Settipani CA, Kendall PC. The Effect of Child Distress on Accommodation of Anxiety: Relations With Maternal Beliefs, Empathy, and Anxiety. J Clin Child Adolesc Psychol 2017 Nov;46(6):810-823. PMID:26672808

20. Shimshoni Y, Silverman WK, Lebowitz ER. SPACE-ARFID: A pilot trial of a novel parent-based treatment for avoidant/restrictive food intake disorder. Int J Eat Disord 2020 Oct;53(10):1623-1635. PMID:33464594

21. Lebowitz ER, Marin C, Martino A, Shimshoni Y, Silverman WK. Parent-Based Treatment as Efficacious as Cognitive-Behavioral Therapy for Childhood Anxiety: A Randomized Noninferiority Study of Supportive Parenting for Anxious Childhood Emotions. J Am Acad Child Adolesc Psychiatry 2020 Mar;59(3):362-372. PMID:30851397

22. Gelinas L, Pierce R, Winkler S, Cohen IG, Lynch HF, Bierer BE. Using Social Media as a Research Recruitment Tool: Ethical Issues and Recommendations. Am J Bioeth 2017 Mar;17(3):3-14. PMID:28207365

23. Kessler RC, Amminger GP, Aguilar-Gaxiola S, Alonso J, Lee S, Ustün TB. Age of onset of mental disorders: a review of recent literature. Curr Opin Psychiatry 2007 Jul;20(4):359- 
364. PMID: 17551351

24. A meta-analysis of parent training: Moderators and follow-up effects. Clin Psychol Rev Pergamon; 2006 Jan 1;26(1):86-104.

25. Tonarely NA, Ehrenreich-May J. Confirming the Factor Structure and Validity of the Distress Tolerance Scale (DTS) in Youth. Child Psychiatry Hum Dev 2020 Aug;51(4):514526. PMID:31637572

26. Ebesutani C, Korathu-Larson P, Nakamura BJ, Higa-McMillan C, Chorpita B. The Revised Child Anxiety and Depression Scale 25-Parent Version: Scale Development and Validation in a School-Based and Clinical Sample. Assessment 2017 Sep;24(6):712-728.

PMID:26834091

27. Meyer TJ, Miller ML, Metzger RL, Borkovec TD. Development and validation of the penn state worry questionnaire [Internet]. Behaviour Research and Therapy. 1990. p. 487-495. [doi: 10.1016/0005-7967(90)90135-6]

28. Schleider JL, Dobias M, Sung J, Mumper E, Mullarkey MC. Acceptability and Utility of an Open-Access, Online Single-Session Intervention Platform for Adolescent Mental Health. JMIR Ment Health 2020 Jun 30;7(6):e20513. PMID:32602846

29. Schleider JL, Mullarkey MC, Weisz JR. Virtual Reality and Web-Based Growth Mindset Interventions for Adolescent Depression: Protocol for a Three-Arm Randomized Trial. JMIR Res Protoc 2019 Jul 9;8(7):e13368. PMID:31290406

30. Schleider JL, Dobias ML, Sung JY, Mullarkey MC. Future Directions in Single-Session Youth Mental Health Interventions. J Clin Child Adolesc Psychol 2020 Mar;49(2):264-278. PMID:31799863

31. Lakens D. Calculating and reporting effect sizes to facilitate cumulative science: a practical primer for t-tests and ANOVAs. Front Psychol [Internet] Frontiers; 2013 [cited 2021 Feb 1];4. [doi: 10.3389/fpsyg.2013.00863]

32. Behar E, Alcaine O, Zuellig AR, Borkovec TD. Screening for generalized anxiety disorder using the Penn State Worry Questionnaire: a receiver operating characteristic analysis. J Behav Ther Exp Psychiatry 2003 Mar;34(1):25-43. PMID:12763391

33. Fleming T, Bavin L, Lucassen M, Stasiak K, Hopkins S, Merry S. Beyond the trial: Systematic review of real-world uptake and engagement with digital self-help interventions for depression, low mood, or anxiety [Internet]. [doi: 10.26686/wgtn.12331259.v1]

34. Rubin DB. Multiple Imputation for Nonresponse in Surveys. John Wiley \& Sons; 2009. ISBN:9780470317365

35. Schleider JL, Mullarkey MC, Chacko A. Harnessing Wise Interventions to Advance the Potency and Reach of Youth Mental Health Services. Clin Child Fam Psychol Rev 2020 Mar;23(1):70-101. PMID:31440858 